\title{
Searches for SUSY in photons and tau channels with the ATLAS detector
}

\author{
Bruce A. Schumm *i \\ Santa Cruz Institute for Particle Physics and the Department of Physics, University of California \\ at Santa Cruz \\ On behalf of the ATLAS Collaboration \\ E-mail: baschumm@ucsc.edu
}

\begin{abstract}
Searches for the prompt decay of supersymmetric particles in events containing photons or taus and large transverse missing momentum, performed by the ATLAS experiment running with $p p$ collisions at $\sqrt{s}=13 \mathrm{TeV}$, are presented. The final states that are considered are motivated by gauge-mediated and supergravity supersymmetry breaking models with stable dark matter candidate as the lightest supersymmetric particle. The increase in the center-of-mass energy of the proton-proton collisions gives a unique opportunity to extend the sensitivity to production of supersymmetric particles at the Large Hadron Collider.
\end{abstract}

38th International Conference on High Energy Physics

3-10 August 2016

Chicago, USA

\footnotetext{
*Speaker.

${ }^{\dagger}$ This work supported in part by the United States Department of Energy, grant DE-SC0010107.
} 


\section{Introduction}

The ATLAS collaboration has performed three supersymmetry (SUSY) inspired searches with $13 \mathrm{TeV} p p$ collisions at the LHC, making use of the ATLAS detector [1] to reconstruct particles and jets arising from the collisions. Signal regions (SRs) optimized for sensitivity to various SUSY scenarios have been defined that include 1) two photons accompanied by significant overall transverse energy (diphoton); 2) one or more photons accompanied by jets and significant transverse energy (photon+jet); and 3) one or more hadronically-decaying $\tau$ leptons and at least one hard jet (tau+jet), all in association with significant missing transverse energy $\left(E_{\mathrm{T}}^{\mathrm{miss}}\right)$.

SUSY introduces a symmetry between fermions and bosons, resulting in a SUSY partner (sparticle) with identical quantum numbers except for a difference of a half-unit of spin from each corresponding Standard Model (SM) particle. The introduction of the symmetry provides a compelling mechanism for the stabilization of the Higgs mass in Grand Unified Theory (GUT) scenarios, and a dark matter candidate that motivates the use of missing transverse energy $E_{\mathrm{T}}^{\text {miss }}$ as a signature for the discovery of SUSY states. Since sparticle states have yet to be observed, SUSY must be a broken symmetry. Several schemes have been put forward to engineer the breaking of SUSY, including 'supergravity' (SUGRA) [2] and 'gauge mediation' (GMSB) [3]. SUGRA models tend to feature a generic 'neutralino' LSP $\left(\tilde{\chi}_{1}^{0}\right)$ composed of an admixture of the bino, neutral wino, and higgsino states. GMSB models feature a light $(<1 \mathrm{GeV})$ gravitino LSP and a stau or $\tilde{\chi}_{1}^{0}$ next-to-lightest sparticle (NLSP); the identity of the NLSP directly impacts the experimental signature that is explored to probe the model.

The tau+jet search is naturally sensitive to GMSB models with a stau NLSP, which can either be produced directly ('electroweak production') or through the decay of a higher-mass gluino state ('strong production'); which of these two processes dominates the potential population of the tau+jet SRs depends on the values of the GMSB parameters associated with the SUSY-breaking scale $(\Lambda)$ and the ratio $\tan \beta$ of the vacuum expectation values of the two SUSY Higgs doublets. The diphoton and photon+jet searches are motivated by GMSB-inspired gauge-mediation models for which SUSY production occurs solely through gluino pairs that each include the $\tilde{\chi}_{1}^{0}$ NLSP in their decay chains; all other SUSY states except the gravitino are assumed to have inaccessibly large mass. For the diphoton search the $\tilde{\chi}_{1}^{0}$ is assumed to be bino-like, with a photonic branching fraction of no less than $\cos \theta_{W}$ for any value of its mass. For the photon+jet search the $\tilde{\chi}_{1}^{0}$ is assumed to be a bino-higgsino admixture, producing a photon or a $Z$ boson in its decay with about equal probability. A final, SUGRA-inspired model is explored with the tau+jet analysis, for which the NLSP is a degenerate triplet of $\tilde{\chi}_{1}^{ \pm}$and $\tilde{\chi}_{2}^{0}$ states with purely stauonic couplings. For this model, production again proceeds through gluino pairs, with the NLSP state masses constrained to be half-way between those of the produced gluino and the stable $\tilde{\chi}_{1}^{0}$ NLSP.

\section{Tau+jet analysis}

Six SRs are defined for the tau+jet analysis: three requiring a single, and three requiring two or more, reconstructed hadronically-decaying $\tau$ leptons. Four regions are optimized for the case for which there is a substantial difference between the production-scale $(\tilde{g})$ and $\operatorname{LSP}\left(\tilde{\chi}_{1}^{0}\right)$ masses, and require significant transverse energy in addition to that of the $\tau$ candidate(s). These include 
the single- and multi- $\tau$ SRs $S R_{\tau}^{L}$ and $S R_{\tau \tau}^{L}$, each targeting gluino masses in the $1300 \mathrm{GeV}$ range in association with a low $(\sim 100 \mathrm{GeV})$ LSP mass. Also included are single- and multi- $\tau$ SRs $S R_{\tau}^{M}$ and $S R_{\tau \tau}^{M}$, optimized for sensitivity to an intermediate $\tilde{g}-\tilde{\chi}_{1}^{0}$ mass splitting in the $500 \mathrm{GeV}$ range. A single- $\tau$ SR $S R_{\tau}^{C}$ targets the 'compressed' region for which this mass difference is below $100 \mathrm{GeV}$, replacing overall transverse-energy requirements with more stringent requirements on $E_{\mathrm{T}}^{\text {miss }}$ as well as the requirement of a stiff jet arising from gluonic initial-state radiation. A final multi- $\tau$ SR $S R_{\tau \tau}^{G}$, optimized for sensitivity to the GMSB model, exploits the high production energy scale of gluinos close to the expected range of sensitivity by requiring a large amount of visible transverse energy. More details on the tau+jet SR definitions can be found in Ref. [4].

Contributions from top and vector boson $(W, Z)$ production comprise the dominant source of SM background, accounting for over $90 \%$ of the expected SM contribution in all but $S R_{\tau \tau}^{G}$, for which they provide over $80 \%$ of the expected SM contribution. The contributions of these processes are estimated from MC simulation, normalized to the population of various control regions (CRs) that target specific SM channels (top, $W, Z$, etc.). Depending on the SM process being constrained, control regions can require or eliminate events with $b$-quark jets and make use of 'fake' $\tau$ leptons identified with relaxed selection criteria. A smaller contribution from multi-jet events is estimated with data-driven techniques described in Ref. [4].

The resulting SM expectations for the six SRs, assuming $3.2 \mathrm{fb}^{-1}$ of $13 \mathrm{TeV} p p$ collision data, is shown in Table 1, along with the number of events observed in each SR. No excess above SM expectation was observed in any of the six SRs, allowing 95\% confidence limits (CLs) to be set on the parameters of the SUGRA and GMSB models. For the SUGRA model, for each point in the $\left(m_{\tilde{g}}, m_{\tilde{\chi}_{1}^{0}}\right)$ plane, the limit was set by the SR providing the lowest expected CL under the assumption of no contribution from SUSY signal. The limits are dominated by the single- $\tau$ analysis in the compressed region and the multi- $\tau$ analysis elsewhere. The resulting limit contours for the two models probed by the tau+jet analysis are exhibited in Figure 1. In the context of SUGRA model, gluino mass limits as high as $1570 \mathrm{GeV}$ are set, although the gluino mass limit degrades significantly in the compressed region.

\section{Diphoton analysis}

Models of gauge mediation featuring a purely bino-like NLSP, which decays to photon + gravitino with a branching fraction of no less than $\cos ^{2} \theta_{W}$, can be sensitively probed with analyses requiring two isolated photons and $E_{\mathrm{T}}^{\mathrm{miss}}$. For a model of gluino production with subsequent decay to the gravitino LSP through a bino-like NLSP, a single SR $\left(S R_{\gamma \gamma}\right)$ was found to provide good sensitivity over the full range $0<m_{\tilde{\chi}_{1}^{0}}<m_{\tilde{g}}$ of bino-like $\tilde{\chi}_{1}^{0}$ mass. In addition to requiring two photons and $E_{\mathrm{T}}^{\text {miss }}$ of at least $175 \mathrm{GeV}$, the analysis exploits the high mass scale associated

Table 1: Summary of numbers of events expected from SM sources, and numbers of observed events, for the six tau+jet SRs. Results shown are for $3.2 \mathrm{fb}^{-1}$ of $13 \mathrm{TeV} p p$ data, and are taken from Ref. [4].

\begin{tabular}{l|c|c|c|c|c|c}
\hline \hline Signature & $S R_{\tau}^{C}$ & $S R_{\tau}^{M}$ & $S R_{\tau}^{L}$ & $S R_{\tau \tau}^{L}$ & $S R_{\tau \tau}^{M}$ & $S R_{\tau \tau}^{G}$ \\
SM Expected & $49.2 \pm 6.2$ & $15.0 \pm 2.4$ & $5.7 \pm 1.2$ & $4.2 \pm 3.0$ & $3.2 \pm 1.2$ & $0.69 \pm 0.24$ \\
Observed & 47 & 11 & 1 & 4 & 0 & 0 \\
\hline \hline
\end{tabular}



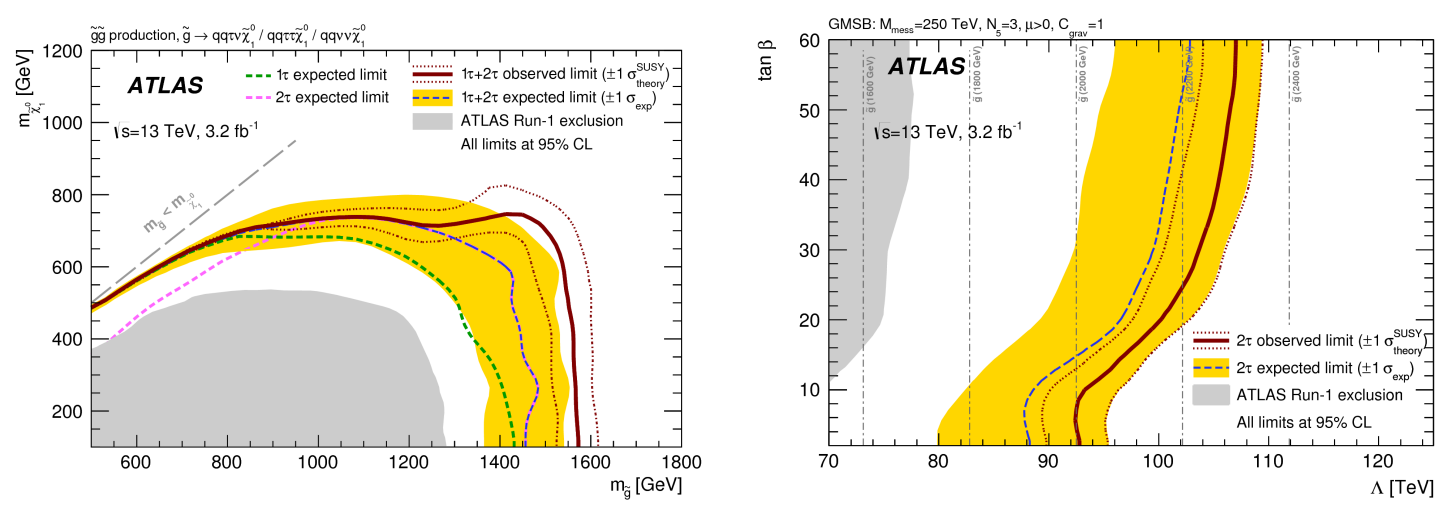

Figure 1: $95 \%$ CL exclusion limits in the $\left(m_{\tilde{g}}, m_{\tilde{\chi}_{1}^{0}}\right)$ plane of the SUGRA (left) and GMSB (right) models explored by the tau+jet analysis, from Ref. [4]. The observed limits are exhibited for the nominal SUSY model cross section, as well as for a SUSY cross section increased and lowered by one standard deviation of the cross-section systematic uncertainty, and make use of the tau+jet SR with the greatest expected sensitivity at the given point in the $\left(m_{\tilde{g}}, m_{\tilde{\chi}_{1}^{0}}\right)$ plane. The dashed green and magenta trajectories represent the limits arising from the $1-\tau$ and $2-\tau$ analyses, respectively.

with gluino production near the expected limit of sensitivity by imposing a requirement that the transverse mass, including the contribution of the reconstructed $E_{\mathrm{T}}^{\text {miss }}$ vector, be at least $1.5 \mathrm{TeV}$.

The dominant SM background contribution to $S R_{\gamma \gamma}$ arises from the production of $W$ bosons in association with two photons, with the $W$ boson decaying leptonically and producing a neutrino that generates missing transverse momentum. The contribution from this background is estimated using MC simulation, scaled to the content of a control region formed by requiring a lepton (electron or muon) in addition to the two photons of $S R_{\gamma \gamma}$. Backgrounds from electrons misidentified as photons are estimated from control samples requiring a single photon and single electron, and scaled by an electron-to-photon misidentification factor determined from electronic $Z$ boson decays. Backgrounds from jets misidentified as photons are estimated by extrapolating from independent sidebands accumulated by weakening the photon identification or isolation requirements.

For an integrated luminosity of $3.2 \mathrm{fb}^{-1}$, the total expected $S M$ contribution to $S R_{\gamma \gamma}$ is estimated to be $0.27_{-0.10}^{+0.22}$ events; for this sample of $13 \mathrm{TeV} p p$ collisions, no events were observed in $S R_{\gamma \gamma}$. Based on the lack of a significant excess relative to the SM background expectation, limits can be set in the $\left(m_{\tilde{g}}, m_{\tilde{\chi}_{1}^{0}}\right)$ plane of the gluino-bino gauge mediation model; these limits are exhibited in Figure 2. Depending on the bino-like $\tilde{\chi}_{1}^{0}$ mass, 95\% CL lower limits between 1650 and 1750 $\mathrm{GeV}$ are set on the gluino mass. Further details of the diphoton analysis are available in Ref. [5].

\section{Photon+jet analysis}

The requirement that selected events need contain no more than a single photon is optimal for gauge-mediation scenarios for which the NLSP $\tilde{\chi}_{1}^{0}$ is not purely bino-like, leading to decays with a smaller photonic branching fraction than for the purely bino-like model constrained with the diphoton analysis. Thus, the photon+jet analysis is optimized for the case of a $\tilde{\chi}_{1}^{0}$ NLSP composed of a bino-higgsino admixture with an approximately $50 \%$ branching fraction to photon+gravitino, independent of the $\tilde{\chi}_{1}^{0}$ mass. The higgsino mass parameter is kept positive, so that the remaining 


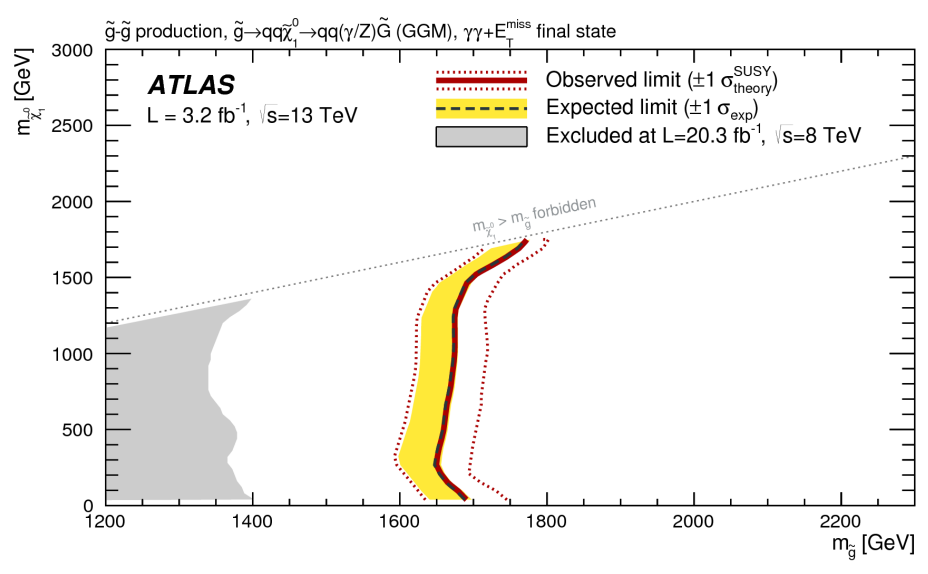

Figure 2: Observed and expected 95\% CL exclusion limits in the $\left(m_{\tilde{g}}, m_{\tilde{\chi}_{1}^{0}}\right)$ mass plane (for a purely binolike $\tilde{\chi}_{1}^{0}$ ) imposed by the diphoton analysis, from Ref. [5]. The observed limits are exhibited for the nominal SUSY model cross section, as well as for a SUSY cross section increased and lowered by one standard deviation of the cross-section systematic uncertainty.

decays are dominated by $Z+$ gravitino, leading to a large number of events with a single photon, one or more jets, and significant missing energy.

Two signal regions are defined for the photon+jet analysis: one each for low $\left(S R_{\gamma}^{L}\right)$ and high $\left(S R_{\gamma}^{H}\right)$ NLSP $\tilde{\chi}_{1}^{0}$ mass relative to that of the produced gluino. To take advantage of the high production mass scale expected at the limit of sensitivity, both photon+jet SRs require $2 \mathrm{TeV}$ of transverse mass, including the contribution of the reconstructed $E_{\mathrm{T}}^{\text {miss }}$ vector, while $S R_{\gamma}^{L}$ exploits the visible activity afforded by the large $m_{\tilde{g}}-m_{\tilde{\chi}_{1}^{0}}$ mass difference by requiring four jets, as opposed to the two required for $S R_{\gamma}^{H}$.

SM backgrounds to the two photon+jet SRs are dominated by the production of photons in association with jets, weak vector bosons ( $W$ or $Z$ ), or top-quark pairs. These backgrounds are estimated via MC simulation, normalized to the content of various control regions targeting each of these processes, defined by requiring lower $E_{\mathrm{T}}^{\text {miss }}$ requirements, applying kinematic selections that enhance sensitivity to weak boson production, and requiring b-quark jets, respectively. Backgrounds arising from the misidentification of electrons and jets are estimated with techniques similar to those used for the diphoton analysis. Further details about the photon+jet SR definition and background estimation are available in Ref. [6].

For an integrated luminosity of $13.3 \mathrm{fb}^{-1}$, the total expected SM contributions to $S R_{\gamma}^{L}$ and $S R_{\gamma}^{H}$ are estimated to be $0.78 \pm 0.18$ and $1.49 \pm 0.45$ events, respectively; for this sample of $13 \mathrm{TeV} p p$ collisions, three events were observed in $S R_{\gamma}^{L}$ and one event in $S R_{\gamma}^{H}$. For $S R_{\gamma}^{L}$, the observation of three events relative to an expected background of $0.78 \pm 0.18$ events has a $2 \%$ likelihood, under the assumption that there is no contribution from physics beyond the SM. Under this assumption, limits can be set in the $\left(m_{\tilde{g}}, m_{\tilde{\chi}_{1}^{0}}\right)$ of the gluino-production higgsino-bino gauge mediation model of the photon+jet analysis; these limits are exhibited in Figure 3. Depending on the bino-like $\tilde{\chi}_{1}^{0}$ mass, 95\% CL lower limits between 1.5 and $2.0 \mathrm{TeV}$ are set on the gluino mass. 


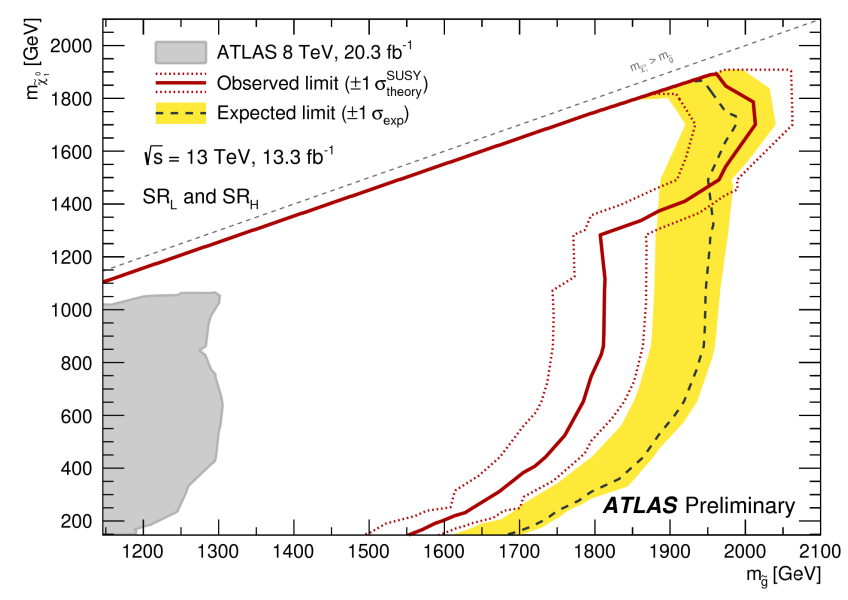

Figure 3: Observed and expected 95\% CL limit in the $\left(m_{\tilde{g}}, m_{\tilde{\chi}_{1}^{0}}\right)$ mass plane of the photon+jet analysis higgsino-bino model, from Ref. [6]. Limits are set using the $S R S R_{\gamma}^{L}$ or $S R_{\gamma}^{L}$ that provides the best expected limit at the given point in the plane. The observed limits are exhibited for the nominal SUSY model cross section, as well as for a SUSY cross section increased and lowered by one standard deviation of the crosssection systematic uncertainty.

\section{Summary and conclusions}

A search for evidence of supersymmetry via the production of $\tau$ leptons or photons in association with missing transverse momentum has been performed with the ATLAS detector, making use of between 3.2 and $13.3 \mathrm{fb}^{-1}$ of $p p$ collision data. No significant excess was observed in any of the defined signal regions, and limits were set within the parameter spaces of several SUGRA and gauge-mediation inspired supersymmetric models. Within the context of these models, limits as high as $2.0 \mathrm{TeV}$ were set on the mass of the gluino, the superpartner of the Standard Model gluon.

\section{References}

[1] ATLAS Collaboration, JINST 3, S08003 (2008).

[2] A. H. Chamseddine, R. L. Arnowitt, and P. Nath, Phys. Rev. Lett. 49, 970 (1982).

[3] M. Dine, W. Fischler, and M. Srednicki, Nucl. Phys. B 189, 575 (1981).

[4] ATLAS Collaboration, "Search for squarks and gluinos in events with hadronically decaying tau leptons, jets and missing transverse momentum in proton-proton collisions at $\sqrt{s}=13 \mathrm{TeV}$ recorded with the ATLAS detector", arXiv:1607.05979 [hep-ex], July 2016; submitted to Eur. Phys. J. C.

[5] ATLAS Collaboration, "Search for supersymmetry in a final state containing two photons and missing transverse momentum in $\sqrt{s}=13 \mathrm{TeV}$ pp collisions at the LHC using the ATLAS detector", arXiv:1606.09150 [hep-ex], June 2016; to appear in Eur. Phys. J. C.

[6] ATLAS Collaboration, "Search for Supersymmetry in events with photons, jets and missing transverse energy with the ATLAS detector in 13 TeV pp collisions", ATLAS-CONF-2016-066, https://cds.cern.ch/record/2206209, August 2016. 